\title{
The Clinical Effect of Zoledronic Acid Combined with Teriparatide in Perverting Recurrent Fracture of Osteoporotic Vertebral Compressive Fractures in the Elderly after Percutaneous Kyphoplasty
}

\author{
Le Zhao ${ }^{1 *}$, Xinming Yang ${ }^{*} \#$ (D), Ying Zhang ${ }^{2}$ \\ ${ }^{1}$ Department of Surgery, Graduate School of Hebei North University, Zhangjiakou, China \\ ${ }^{2}$ Department of Orthopaedics, The First Affiliated Hospital of Hebei North University, Zhangjiakou, China \\ Email: "yxm11200@126.com
}

How to cite this paper: Zhao, L., Yang, X.M. and Zhang, Y. (2021) The Clinical Effect of Zoledronic Acid Combined with Teriparatide in Perverting Recurrent Fracture of Osteoporotic Vertebral Compressive Fractures in the Elderly after Percutaneous Kyphoplasty. Surgical Science, 12, 161-173.

https://doi.org/10.4236/ss.2021.126018

Received: March 16, 2021

Accepted: May 31, 2021

Published: June 3, 2021

Copyright $\odot 2021$ by author(s) and Scientific Research Publishing Inc. This work is licensed under the Creative Commons Attribution International License (CC BY 4.0).

http://creativecommons.org/licenses/by/4.0/

\begin{abstract}
Background: Zoledronic acid and teriparatide have been proved to be effective in improving bone metabolism and preventing fractures, but there is no clear clinical report on the efficacy of their combined application. Purpose: To discuss the clinical effect of zoledronic acid combined with teriparatide in perverting recurrent fracture of osteoporotic vertebral compressive fractures (OVCF) in the elderly after percutaneous kyphoplasty (PKP). Method: A randomized clinical trial was conducted at the First Affiliated Hospital of Hebei North University in China from September 2018 and September 2019. A total of 60 patients with OVCF were enrolled in the study (zoledronic acid: 20 cases; teriparatide: 20 cases; zoledronic acid + teriparatide: 20 cases). Observe and compare the changes of bone mineral density (BMD), pro-collagen type I N-terminal propeptide (PINP) and cross-linked C-terminal telopeptide of type I collagen ( $\beta$-CTX) before surgery, 6 months and 1 year after surgery. At the same time, secondary fracture events and adverse reaction events were recorded during the follow-up period. Results: After normalized treatment, the bone metabolism indexes of PINP and $\beta$-CTX were improved and BMD was increased in three groups. Adverse Reactions: There was no statistical significance in the incidence of fever, gastrointestinal reactions and myalgia among the three groups $(\mathrm{P}>0.05)$. The incidence of recurrent fractures in group $\mathrm{A}$ was higher than that in group $\mathrm{C}(\mathrm{P}<0.05)$, but there was no significant difference between group $B$ and group $C(P>0.05)$. Conclusion: Zoledronic
\end{abstract}

${ }^{\star}$ Co-first authors

${ }^{*}$ Corresponding author. 
acid combined with teriparatide is superior to Zoledronic acid in preventing the risk of recurrent fracture after PKP for old patients with OVCF, but it has no significant advantage over teriparatide.

\section{Keywords}

Zoledronic Acid, Teriparatide, Percutaneous Kyphoplasty (PKP),

Osteoporotic Vertebral Compressive Fractures (OVCF)

\section{Introduction}

According to statistics, the number of people over 65 who suffer from osteoporosis in China is as high as 144 million, and the number of patients with fracture caused by osteoporosis (OP) is increasing year by year. About $50 \%$ of OP fractures in the elderly occur in the spine, especially in the thoracolumbar spine [1] [2] [3]. Osteoporotic vertebral compressive fractures (OVCF) has become an urgent public health problem. Since the introduction of percutaneous kyphoplasty/vertebroplasty (PKP/PVP) into China in the 1980s, they have gradually developed and become the main surgical treatment for OVCF [4] [5]. Epidemiological investigation shows that the rate of recurrent fracture after PKP is as high as $25 \%$, which is mainly related to the non-standardized treatment of OP [6] [7]. To find appropriate anti-OP medicines is the key to prevent the recurrence of OVCF after PKP.

At present, anti-OP medicines mainly include anti-resorption medicines and promoting bone formation medicines [8] [9]. Zoledronic acid is an anti-resorption drugs commonly used in clinical practice. It has been approved for the treatment of primary or secondary osteoporosis all over the world (approved indications vary between countries) [10]. The conclusion was reported by Smith, D. L. and others that zoledronic acid can improve BMD and reduce the risk of fracture in a 6-year randomized and double-blind trial about 2000 women over 65 with OP [8]. Teriparatide is commonly used as a medicine to promote bone formation in clinical practice, which has significant effect in improving OP and preventing osteoporotic fracture, but there is no clear evidence to show that it can promote fracture healing [9]. Clinical studies have shown that zoledronic acid combined with teriparatide may weaken the anabolism of teriparatide. The conclusion was reported by Cosman, F. and others that the combined use of zoledronic acid combined with teriparatide can improve bone metabolism, increase BMD, and reduce fracture risk to the greatest extent in a double-blind, randomized, multicenter and multinational study on 412 postmenopausal women with OP (mean age $65 \pm 9$ years) [11]. However, in this study, the fracture risk base of postmenopausal women with OP has not been unified. There is a lack of stronger evidence in the treatment of osteoporotic fracture. In a recent study on the risk of recurrent fractures in diabetic patients with OVCF, zoledronic acid combined with teriparatide can significantly reduce the rate of re- 
current fractures compared with zoledronic acid after PKP [12]. This study is to observe and compare the clinical effect of zoledronic acid, teriparatide, zoledronic acid combined with teriparatide in the prevention of recurrent fracture risk in elderly patients with OVCF after PKP, so as to further unify the data of fracture risk base, and find a better treatment scheme for elderly patients with OVCF.

\section{Materials and Methods}

\subsection{Cases Data}

This retrospective clinical data analysis collected 60 patients with OVCF hospitalized in the orthopedics department in the First Affiliated Hospital of Hebei North University in China from September 2018 to September 2019. The study was approved by the medical ethics committee of the First Affiliated Hospital of Hebei North University.

Inclusion criteria: 1) Age $\geq 65$; 2) Preoperative BMD T value $\leq-2.5 \mathrm{SD}$; 3 ) Diagnosis of osteoporotic single vertebral $\left(\mathrm{T}_{5}-\mathrm{L}_{5}\right)$ compressive fractures, fracture stability without spinal cord and redundant nerve damage, and the patient's medical history $\leq 2$ weeks; 4) Patients who had not used relevant medicine such as zoledronic acid or teriparatide continuously for more than 3 months (short-term users, who could be enrolled after a 1-year washout period); 5) All had received surgical treatment for PKP; 6) They were followed for at least 1 year.

Exclusion criteria: 1) Patients with non-primary OVCF; 2) Patients with other pathological compression fractures of the thoracolumbar spine due to bone metastasis of malignancy; 3) Patients with long-term systemic use of glucocorticoids in the previous 1 year and prior treatment with oestrogen, raloxifene or calcitonin within 3 months.

\subsection{Sample Size Estimation}

SPSS20.0 statistical software was used to analyze the sample size required in the study. The presupposed medium effect size was $\mathrm{f}=0.25$ (Cohen, 1988), the statistical power was $1-\beta=0.8$, and the significance level was $\alpha=0.05$. The results showed that at least 53 patients were needed in each group. Because it was a single centre study and time constraints, a total of 60 patients were taken conveniently for this study. They were divided into zoledronic acid (group A, $n=20$ ), Teriparatide (group B, $\mathrm{n}=20$ ) and zoledronic acid + Teriparatide (Group C, $\mathrm{n}=$ 20).

\subsection{Experimental Design}

\subsubsection{Preoperative Preparation}

The relevant inspection results were completed before surgery. Deal with other combined injuries, and stabilize cardiovascular, cerebrovascular, endocrine and other basic diseases. 


\subsubsection{Surgical Methods}

The surgery was completed by two associate chief physicians. Assist the patients to prone position, abdominal suspension, C-arm fluoroscopy, and body surface marking the diseased vertebrae. Routinely sterilize, spread towel, and local anesthesia. A bilateral approach was taken and the diseased vertebrae were accessed percutaneously using a puncture trocar under C-arm fluoroscopy, along the bilateral pedicles. The inner core of the puncture needle was replaced with a guidewire so that it was $2-3 \mathrm{~mm}$ anterior to the cortex at the posterior border of the diseased vertebrae. The working cannula was changed, and iodixanol was infused intrasaccular to dilate the balloon to the upper and lower endplates to restore a satisfactory vertebral height. The contrast medium was withdrawn, the balloon removed, and the bone cement filled to completely fill the cavity. The injection device was extracted after cement had solidified. All patients were given $12 \mathrm{~h}-24 \mathrm{~h}$ ECG monitoring after surgery. At the same time, the sensorimotor function of both lower limbs of the patient was observed, and moderate activity under the brace was worn $24 \mathrm{~h}$ after surgery.

\subsection{Medication and Grouping}

\subsubsection{Basic Pharmacological Interventions}

All patients were given calcium carbonate D3 after surgery.

\subsubsection{Therapeutic Medicine Intervention}

Group A: intravenous infusion zoledronic acid $5 \mathrm{mg}$ ( 1 time in 1 year); group B: hypodermic injection teriparatide $20 \mathrm{~g} / \mathrm{d}$ for 1-year continuous treatment; group C: intravenous infusion zoledronic acid $5 \mathrm{mg}$ ( 1 time in 1 year) followed by hypodermic injection teriparatide $20 \mathrm{~g} / \mathrm{d}$ for 1 -year continuous treatment.

Zoledronic acid Injection (trade name: yigu, specification: $5 \mathrm{mg} / \mathrm{branch}$, Zhengdatianqing Co.Ltd, product batch: National Standard No H20113138); Teriparatide Injection $20 \mathrm{~g}$ (trade name: futaiao, specification: $20 \mathrm{~g} / \mathrm{branch}$, Lilly France Pharmaceutical Co. Ltd, product batch: National Standard No: A865645C).

\subsection{Observation Indexes}

\subsubsection{Base Data}

The data of age, gender and vertebral distribution were recorded.

\subsubsection{Data before Treatment}

The data of BMD, pro-collagen type I N-terminal propeptide (PINP) and cross-linked C-terminal telopeptide of type I collagen ( $\beta$-CTX) were observed and recorded before treatment.

\subsubsection{Data after Treatment}

The data of BMD, PINP and $\beta$-CTX were observed and recorded at 6 months and 1 year after the standardized treatment in each group. The data of fever, gastrointestinal reaction, myalgia and recurrent thoracolumbar fractures were recorded during the follow-up period. 


\subsection{Statistical Analysis}

SPSS 20.0 statistical software was used to analyze the data of the 3 groups. The data of the 3 groups were consistent with the normal distribution and the homogeneity of variance. The comparison between the groups was performed by analysis of variance (ANOVA). If there were differences between the groups, the LSD-t test was used. The comparison between the groups was performed by paired t-test. $\mathrm{P}<0.05$ was regarded as the difference with statistical significance.

\section{Results}

\subsection{Analysis of Patients' Baseline Data}

60 cases were enrolled in the experiment, 20 cases in group A, 20 cases group B and 20 cases in group $C$. There were no significant differences in age, gender and distribution of diseased vertebrae among the three groups $(\mathrm{P}>0.05)$ (Table 1$)$. There were no significant differences in BMD, PINP and $\beta$-CTX before treatment $(\mathrm{P}>0.05)$ (Table 2). There were no lost cases, missed follow-up cases and death cases during the follow-up period.

\subsection{BMD}

BMD in the 3 groups increased at 6 months after treatment, but the differences were not statistically significant $(\mathrm{P}>0.05)$ (Table 3$)$. And there was no significant difference between the 3 groups $(\mathrm{F}=3.93, \mathrm{P}=0.26)$. Compared to pre-treatment, BMD in the 3 groups increased significantly at 1 year after treatment $(\mathrm{P}<0.05)$ (Table 3). And there was significant difference between the 3 groups $(F=6.79, P<0.05)$. In terms of improving $B M D$, group $C$ was better than group B, and group B was better than group A (Table 4).

Table 1. Comparison of the OVCF baseline information.

\begin{tabular}{ccccc}
\hline Item & $\begin{array}{c}\text { Sex } \\
(\mathrm{M} / \mathrm{F})\end{array}$ & $\begin{array}{c}\text { Age } \\
(\text { year })\end{array}$ & $\begin{array}{c}\text { diseased vertebrae } \\
\left(\mathrm{T}_{5-10} / \mathrm{T}_{11}-\mathrm{L}_{1} / \mathrm{L}_{2-5}\right)\end{array}$ & $\begin{array}{c}\text { All } \\
(\text { case })\end{array}$ \\
\hline $\mathrm{A}$ & $8 / 12$ & $73.57 \pm 3.47$ & $3 / 12 / 5$ & 20 \\
$\mathrm{~B}$ & $10 / 10$ & $74.13 \pm 2.94$ & $2 / 15 / 3$ & 20 \\
$\mathrm{C}$ & $7 / 13$ & $74.53 \pm 3.70$ & $4 / 10 / 6$ & 20 \\
$\mathrm{~F} / \mathrm{X}^{2}$ & 0.27 & 0.62 & 0.77 & \\
$\mathrm{P}$ & 0.87 & 0.57 & 0.94 & \\
\hline
\end{tabular}

Table 2. Comparison of the information of pre-treatment.

\begin{tabular}{ccccc}
\hline Item & BMD (SD) & PINP $(\mathrm{g} / \mathrm{L})$ & $\beta$-CTX $(\mathrm{g} / \mathrm{L})$ & All (case) \\
\hline A & $-2.96 \pm 0.18$ & $58.57 \pm 1.68$ & $0.55 \pm 0.32$ & 20 \\
B & $-2.94 \pm 0.22$ & $59.76 \pm 1.83$ & $0.53 \pm 0.19$ & 20 \\
C & $-2.96 \pm 0.19$ & $58.85 \pm 1.83$ & $0.54 \pm 0.24$ & 20 \\
F & 0.11 & 2.62 & 2.68 & \\
P & 0.90 & 0.07 & 0.06 & \\
\hline
\end{tabular}


Table 3. Comparison of the OVCF information after treatment.

\begin{tabular}{ccccccc}
\hline Item & Group & Pre-treatment & $\begin{array}{c}6 \text { months } \\
\text { pro-treatment }\end{array}$ & $\begin{array}{c}1 \text { year } \\
\text { pro-treatment }\end{array}$ & $\mathrm{t}$ & $\mathrm{P}$ \\
\hline BMD & A & $-2.96 \pm 0.18$ & $-2.94 \pm 0.17^{\mathrm{a}}$ & $-2.62 \pm 0.20^{\mathrm{b}}$ & $-1.45 /-8.52$ & $0.16^{\mathrm{a}} /<0.05^{\mathrm{b}}$ \\
(SD) & B & $-2.94 \pm 0.22$ & $-2.90 \pm 0.23^{\mathrm{a}}$ & $-2.32 \pm 0.19^{\mathrm{b}}$ & $-1.83 /-0.29$ & $0.08^{\mathrm{a}} /<0.05^{\mathrm{b}}$ \\
& C & $-2.96 \pm 0.19$ & $-2.92 \pm 0.20^{\mathrm{a}}$ & $-2.06 \pm 0.23^{\mathrm{b}}$ & $-1.93 /-9.80$ & $0.06^{\mathrm{a}} /<0.05^{\mathrm{b}}$ \\
PINP & A & $58.57 \pm 1.68$ & $44.83 \pm 12.35^{\mathrm{a}}$ & $43.10 \pm 10.62^{\mathrm{b}}$ & $-12.38 /-13.42$ & $<0.05^{\mathrm{a}} /<0.05^{\mathrm{b}}$ \\
(g/L) & B & $59.76 \pm 1.83$ & $72.34 \pm 15.16^{\mathrm{a}}$ & $68.73 \pm 13.72^{\mathrm{b}}$ & $9.89 / 5.34$ & $<0.05^{\mathrm{a}} /<0.05^{\mathrm{b}}$ \\
& C & $58.85 \pm 1.83$ & $65.74 \pm 8.98^{\mathrm{a}}$ & $64.23 \pm 14.61^{\mathrm{b}}$ & $6.72 / 5.89$ & $<0.05^{\mathrm{a}} /<0.05^{\mathrm{b}}$ \\
$\beta-$ & A & $0.55 \pm 0.32$ & $0.29 \pm 0.08^{\mathrm{a}}$ & $0.26 \pm 0.13^{\mathrm{b}}$ & $-0.32 /-0.35$ & $<0.05^{\mathrm{a}} /<0.05^{\mathrm{b}}$ \\
CTX & B & $0.53 \pm 0.19$ & $0.43 \pm 0.12^{\mathrm{a}}$ & $0.38 \pm 0.21^{\mathrm{b}}$ & $-0.13 /-0.16$ & $<0.05^{\mathrm{a}} /<0.05^{\mathrm{b}}$ \\
(g/L) & C & $0.54 \pm 0.24$ & $0.39 \pm 0.31^{\mathrm{a}}$ & $0.32 \pm 0.14^{\mathrm{b}}$ & $-0.19 /-0.21$ & $<0.05^{\mathrm{a}} /<0.05^{\mathrm{b}}$ \\
\hline
\end{tabular}

Note: a: comparison between 6 months pro-treatment and per-treatment; b comparison between 1 year pro-treatment and per-treatment.

Table 4. Intercomparison of 3 groups at 6 months and 1 year after treatment.

\begin{tabular}{cccc}
\hline Item & A, B/B, A & A, C/C, A & B, C/C, B \\
\hline BMD of 6 months pro-treatment & 0.35 & 0.14 & 0.42 \\
BMD of 1 year pro-treatment & $<0.05$ & $<0.05$ & $<0.05$ \\
& $<0.05$ & $<0.05$ & $<0.05$ \\
PINP of 6 months pro-treatment & $<0.05$ & $<0.05$ & $<0.05$ \\
PINP of 1 year pro-treatment & $<0.05$ & $<0.05$ & $<0.05$ \\
$\beta$-CTX of 6 months pro-treatment & $<0.05$ & $<0.05$ & $<0.05$ \\
B-CTX of 1year pro-treatment & & & \\
\hline
\end{tabular}

\subsection{PINP}

The PINP of group A at 6 months and 1 year after treatment was lower than that before treatment, and the difference was statistically significant $(\mathrm{P}<0.05)$. The PINP of group B and group $C$ at 6 months and 1 year after treatment was higher than that before treatment, and the difference was statistically significant $(\mathrm{P}<$ 0.05 ) (Table 3). There was significant difference among the 3 groups at 6 months after treatment $(\mathrm{F}=9.26, \mathrm{P}<0.05)$. In terms of improving PINP, the PINP of group B was better than that of group C and the PINP of group C was better than that of group A at 6 months after treatment. There was significant difference among the 3 groups at 1 year after treatment $(\mathrm{F}=8.48, \mathrm{P}<0.05)$. In terms of improving PINP, the PINP of group $\mathrm{B}$ was better than that of group $\mathrm{C}$ and the PINP of group C was better than that of group A at 1 year after treatment (Table 4).

\section{4. $\beta$-CTX}

Compared to pre-treatment, $\beta$-CTX in the 3 groups increased at 6 months and 1 year after treatment $(\mathrm{P}<0.05)$ (Table 3$)$. There was significant difference among the 3 groups at 6 months after treatment $(\mathrm{F}=6.38, \mathrm{P}<0.05)$. In terms of im- 
proving $\beta$-CTX, the $\beta$-CTX of group A was better than that of group C and the $\beta$-CTX of group C was better than that of group B at 6 months after treatment. There was significant difference among the 3 groups at 1year after treatment ( $\mathrm{F}$ $=7.26, \mathrm{P}<0.05)$. In terms of improving $\beta$-CTX, the $\beta$-CTX of group $\mathrm{A}$ was better than that of group $\mathrm{C}$ and the $\beta$-CTX of group $\mathrm{C}$ was better than that of group $B$ at 1 year after treatment (Table 4).

\subsection{Adverse Events during the Follow-Up Period}

There were no significant differences in the incidence of fever, gastrointestinal reactions and myalgia among the 3 groups $(\mathrm{P}>0.05)$. And the incidence of recurrent fracture was significantly different $(\mathrm{P}<0.05)$. It was higher in group $\mathrm{A}$ than in group $\mathrm{B}$ and group $\mathrm{C}(\mathrm{t}=4.33, \mathrm{P}=0.04 ; \mathrm{t}=7.06, \mathrm{P}=0.01)$, and there was no significant different between group $\mathrm{B}$ and group $\mathrm{C}(\mathrm{t}=1.03, \mathrm{P}=0.50)$ (Table 5).

\section{Discussion}

\subsection{Clinical Characteristics of OVCF}

About $50 \%$ of OP fractures occurs in the spine, and the thoracolumbar spine is the most common [2] [13]. From the perspective of mechanics and biology, experts, like Ren, think that the probability of thoracolumbar vertebral compression fracture is higher, and patients with thoracolumbar vertebral fracture have higher risk than other vertebral fractures under the same clinical intervention after operation [7]. It is consistent with the data of this study $(37 / 60,61.67 \%)$. Research data showed that the vast majority of OVCF patients (44/60, 73.33\%) were not definitely diagnosed as OP before admission. The discovery of OP mostly originated from the first fracture caused by OP [3] [6]. Microscopically, OP refers to the decrease of normal mineralized bone mass per unit volume, and the parallelism decrease of bone matrix and mineral salts [2] [5] [13]. In 2001, the National Institutes of Health (NIH) proposed a macro definition of OP, which emphasizes the decrease of bone strength and the increase of fracture risk, that is, OP not only reduces the amount of phalangeal bone, but also decreases the mechanical properties [1] [13]. Regular monitoring of BMD, assessment of fracture risk and active intervention in the process of $\mathrm{OP}$ can effectively reduce

Table 5. Comparison of adverse events in 3 groups.

\begin{tabular}{ccccc}
\hline Item & Fever & Gastrointestinal reaction & Myalgia & New fractures \\
\hline A & 3 & 1 & 2 & 6 \\
B & 1 & 0 & 1 & 1 \\
$\mathrm{C}$ & 4 & 1 & 2 & 0 \\
$\mathrm{X}^{2}$ & 2.02 & 1.03 & 0.44 & 10.03 \\
$\mathrm{P}$ & 0.36 & 0.60 & 0.80 & $<0.05$
\end{tabular}


the prevalence of OVCF [13]. The data of this study showed that there were 35 female patients $(35 / 60,58.33 \%)$ in OVCF patients, which was lower than that of large sample data [3] [4]. It was related to the selection of patients over 65. With the growth of age, postmenopausal women with primary osteoporosis enter the elderly primary osteoporosis after about $5-10$ years, that is, the majority of OVCF patients over 65 belong to the elderly OVCF [13]. Therefore, the gender difference is not obvious. At the same time, OP patients over 65 belong to low transformation type, which can eliminate the bias of different bone metabolism types in clinical data observation.

\subsection{The Diagnosis of $\mathrm{OP}$}

At present, the diagnosis of OP mostly depends on the clinical symptoms and BMD examination [13] [14] [15]. BMD can reflect 70\% of the bone mass, which is the gold standard for the diagnosis of OP [4]. However, the value change of BMD has a lag period of $6-12$ months, and the sensitivity is low, which cannot provide effective help for the early diagnosis of OP [16] [17]. At the same time, BDM cannot reflect the process of bone turnover and bone metabolism, and it cannot provide evaluation for the short-term efficacy of clinical medication [17] [18]. Therefore, it cannot provide timely help for the selection of clinical medication. In this study, there was no significant difference in BMD at 6 months after treatment, but significant differences in PINP and $\beta$-CTX can be seen. PINP and $\beta$-CTX are the latest clinical indicators of bone metabolism [19]. Compared with BMD, PINP and $\beta$-CTX have better specificity and is the markers of bone formation and bone resorption [20] [21]. They are of great significance in evaluating OP, preventing and treating OVCF. PINP is expressed and released by activated osteoblasts, which is a sensitive indicator of bone formation [22]. The International Symposium on osteoporosis suggested that PINP should be used as a specific and sensitive indicator of bone formation [23]. $\beta$-CTX is a sensitive indicator of bone resorption. Relevant clinical studies have shown that $\beta$-CTX can be used to monitor the timeliness and efficacy of treatment in patients with osteoporosis [24]. This study shows that although zoledronic acid combined with teriparatide in the prevention of recurrent fractures has no significant difference with teriparatide monotherapy group, its improvement in BMD, $\beta$-CTX is still better than teriparatide monotherapy group. The follow-up time can further extend to observe and count the rate of recurrent fractures, which aims to draw the final conclusion.

\subsection{The Risk Factors of Recurrent Fractures after PKP}

Clinical data shows that the rate of recurrent fracture after PKP is as high as $25 \%$. If the patients do not receive standardized anti-OP treatment within 3 years, the rate of recurrent fracture will reach 50\% [25] [26]. At the same time, the recurrent fracture can further aggravate the damage of centrum and affect the prognosis of OVCF, which is a problem that OVCF patients must face [27]. 
At present, it is believed that it is closely related to stress increases of adjacent vertebrae which cause by bone cement strengthening the hardness of diseased vertebrae, and it is also related to ineffective treatment [5] [27]. The latest view is that recurrent fracture after OVCF is one of the manifestations of OP's natural progress [28]. Therefore, ineffective treatment for OP is an important risk factor for fracture recurrence after PKP in OVCF patients. In this clinical study, there was no statistically significant difference in the distribution of diseased vertebrae in each group. PKP surgery can restore the height of diseased vertebrae as much as possible. Meanwhile, single diseased vertebrae and bilateral approaches are selected to make the distribution of bone cement in centrum satisfactory and as similar as possible. And the bias caused by poor recovery of vertebral height and different degree of bone cement enhancement is excluded. The data showed that the recurrent fracture rate $(7 / 60,11.67 \%)$ was lower than the literature data, which may be related to the standardized anti-OP treatment in this study [5] [13]. And it indirectly proved that the natural process of OP is an important reason of recurrent fracture.

\subsection{Zoledronic Acid Combined with Teriparatide in the Prevention of Recurrent Fracture after PKP}

Zoledronic acid can inhibit the activity of osteoclasts through antagonistic effect to inhibit bone absorption and reduce the incidence of fracture. It has strong adsorption, and low medicine loss rate [29] [30]. The medicine can be absorbed by bone tissue again. The medicine efficacy cycle is long, so it only needs to be injected once a year. The patient's compliance is high [10] [31]. Teriparatide is an active fragment of parathyroid hormone, and it is an inhibitor of bone resorption [32] [33]. It can promote bone formation, improve BDM and reduce the risk of fracture [34]. At the same time, it has been clinically proved that it can be used not only for the treatment of osteoporosis, but also for promoting fracture healing [35]. Clinical data shows that Teriparatide is superior to zoledronic acid in improving BMD in OP patients. The sequential therapy based on the bone remodeling of physiological cycle has not achieved good clinical effica$c y$, even it is not better than the single use of anti-resorption medicine [36] [37]. Clinical data shows that there is no significant difference in adverse reactions between multiple anti-OP medicine combined with sequential therapy, which can produce greater superposition and synergistic effect, but the best combined sequential therapy has not been determined yet [37] [38]. Zoledronic acid combined with teriparatide may weaken the anabolism of teriparatide. Relevant clinical studies have shown that zoledronic acid combined with teriparatide has no obvious advantage over teriparatide in increasing BMD [39]. However, in an experiment of zoledronic acid combined with teriparatide after lumbar spinal fusion in ovariectomized rats, it can significantly improve the quality of bone fusion, and it is clinically proved to be effective [9]. At the same time, studies have shown that zoledronic acid combined with teriparatide can significantly reduce the rate of recurrent fractures compared with zoledronic acid [8]. Although the 
combined use in this clinical study has excellent performance in improving $\mathrm{BMD}$, it cannot effectively reduce the incidence of recurrent fractures. It may be related to the smaller data sample and shorter follow-up time. Meanwhile, the research shows that the choice of OP treatment medicine should be based on bone metabolic markers [24] [40]. Low conversion medicine are mainly promoting bone metabolism, while high conversion medicine are mainly inhibiting bone resorption [40]. The results show that zoledronic acid combined with teriparatide is better than the single medicine group in improving bone metabolism markers PINP and $\beta$-CTX. However, the follow-up time is only one year, which may cover up some data.

\section{Conclusion}

Zoledronic acid combined with teriparatide has no significant increase in clinical adverse reactions compared with single drug group. Zoledronic acid combined with teriparatide can promote bone formation and has clear anti-resorption effect. The combination is better than zoledronic acid in preventing the risk of recurrent fracture after PKP of senile OVCF, but it has no obvious advantage over teriparatide.

\section{Limitations}

The number of clinical cases in this experiment is small, which cannot provide more accurate data support. Relevant clinical workers can take a multi-center study and select more cases to get more convincing data. In this study, we selected cases over 65 years old, which belong to the condition of low bone transformation. Whether it is suitable for the condition of high bone transformation at the same time, it remains to be further verified by clinical trials. The follow-up time of this study is short, but the current clinical medicine treatment cycle is 3 years for zoledronic acid and 2 years for teriparatide, which can further extend the follow-up time to get the final conclusion.

\section{Acknowledgements}

Foundation items: Medical research project of Hebei Provincial Health Commission in 2021; Zhangjiakou High-level Innovation Team.

\section{Conflicts of Interest}

The authors declare no conflicts of interest regarding the publication of this paper.

\section{References}

[1] Cavalier, E., Lukas, P., Bottani, M., et al. (2020) European Biological Variation Study (EuBIVAS): Within- and Between-Subject Biological Variation Estimates of $\beta$-Isomerized C-Terminal Telopeptide of Type I Collagen ( $\beta$-CTX), N-Terminal Propeptide of Type I Collagen (PINP), Osteocalcin, Intact Fibroblast Growth Factor 23 and Uncarboxylated-Unphosphorylated Matrix-Gla Protein-A Cooperation be- 
tween the EFLM Working Group on Biological Variation and the International Osteoporosis Foundation-International Federation of Clinical Chemistry Committee on Bone Metabolism. Osteoporosis International, 31, 1461-1470.

https://doi.org/10.1007/s00198-020-05362-8

[2] Cheng, C., Wentworth, K. and Shoback, D.M. (2020) New Frontiers in Osteoporosis Therapy. Annual Review of Medicine, 71, 277-288. https://doi.org/10.1146/annurev-med-052218-020620

[3] Wang, F., Wang, L.F., Miao, D.C., et al. (2018) Which One Is More Effective for the Treatment of Very Severe Osteoporotic Vertebral Compression Fractures: PVP or PKP? Journal of Pain Research, 11, 2625-2631. https://doi.org/10.2147/JPR.S179022

[4] Yang, H., Liu, H., Wang, S., et al. (2016) Review of Percutaneous Kyphoplasty in China. Spine (Phila Pa 1976), 41, B52-B58. https://doi.org/10.1097/BRS.0000000000001804

[5] Zuo, X.H., Zhu, X.P., Bao, H.G., et al. (2018) Network Meta-Analysis of Percutaneous Vertebroplasty, Percutaneous Kyphoplasty, Nerve Block, and Conservative Treatment for Nonsurgery Options of Acute/Subacute and Chronic Osteoporotic Vertebral Compression Fractures (OVCFs) in Short-Term and Long-Term Effects. Medicine (Baltimore), 97, e11544. https://doi.org/10.1097/MD.0000000000011544

[6] Li, H.M., Zhang, R.J., Gao, H., et al. (2018) New Vertebral Fractures after Osteoporotic Vertebral Compression Fracture between Balloon Kyphoplasty and Nonsurgical Treatment PRISMA. Medicine (Baltimore), 97, e12666. https://doi.org/10.1097/MD.0000000000012666

[7] Ren, H.-L., Jiang, J.-M., Chen, J.-T., et al. (2015) Risk Factors of New Symptomatic Vertebral Compression Fractures in Osteoporotic Patients Undergone Percutaneous Vertebroplasty. European Spine Journal, 24, 750-758.

[8] Reid, I.R., Horne, A.M., Mihov, B., et al. (2019) Anti-Fracture Efficacy of Zoledronate in Subgroups of Osteopenic Postmenopausal Women: Secondary Analysis of a Randomized Controlled Trial. Journal of Internal Medicine, 286, 221-229. https://doi.org/10.1111/joim.12901

[9] Yishake, M., Yasen, M., Jiang, L., et al. (2018) Effects of Combined Teriparatide and Zoledronic Acid on Posterior Lumbar Vertebral Fusion in an Aged Ovariectomized Rat Model of Osteopenia. Journal of Orthopaedic Research, 36, 937-944. https://doi.org/10.1002/jor.23682

[10] Dhillon, S. (2016) Zoledronic Acid (Reclast ${ }^{\oplus}$, Aclasta ${ }^{\varpi}$ ): A Review in Osteoporosis. Drugs, 76, 1683-1697. https://doi.org/10.1007/s40265-016-0662-4

[11] Cosman, F., Eriksen, E.F., Recknor, C., et al. (2011) Effects of Intravenous Zoledronic Acid plus Subcutaneous Teriparatide [rhPTH(1-34)] in Postmenopausal Osteoporosis. Journal of Bone and Mineral Research, 26, 503-511. https://doi.org/10.1002/jbmr.238

[12] Zhang, J., Yan, B., Chen, Z., et al. (2020) Risk of New Vertebral Fracture and Combination Therapy with Zoledronic Acid and Teriparatide in Diabetic Patients after Percutaneous Kyphoplasty. Asian Spine Journal. https://doi.org/10.31616/asj.2020.0282

[13] Mazziotti, G., Bilezikian, J., Canalis, E., et al. (2012) New Understanding and Treatments for Osteoporosis. Endocrine, 41, 58-69. https://doi.org/10.1007/s12020-011-9570-2

[14] Hiligsmann, M., Evers, S.M., Ben Sedrine, W., et al. (2015) A Systematic Review of Cost-Effectiveness Analyses of Drugs for Postmenopausal Osteoporosis. Pharmacoeconomics, 33, 205-224. https://doi.org/10.1007/s40273-014-0231-1 
[15] Szulc, P. (2018) Bone Turnover: Biology and Assessment Tools. Best Practice \& Research Clinical Endocrinology \& Metabolism, 32, 725-738.

https://doi.org/10.1016/j.beem.2018.05.003

[16] Berry, S.D., Dufour, A.B., Travison, T.G., et al. (2018) Changes in Bone Mineral Density (BMD): A Longitudinal Study of Osteoporosis Patients in the Real-World Setting. Archives of Osteoporosis, 13, 124. https://doi.org/10.1007/s11657-018-0528-3

[17] Kapuš, O., Gába, A. and Lehnert, M. (2020) Relationships between Bone Mineral Density, Body Composition, and Isokinetic Strength in Postmenopausal Women. Bone Reports, 12, Article ID: 100255. https://doi.org/10.1016/j.bonr.2020.100255

[18] Johansson, H., Kanis, J.A., Oden, A., et al. (2009) BMD, Clinical Risk Factors and Their Combination for Hip Fracture Prevention. Osteoporosis International, 20, 1675-1682. https://doi.org/10.1007/s00198-009-0845-X

[19] Højsager, F.D., Rand, M.S., Pedersen, S.B., et al. (2019) Fracture-Induced Changes in Biomarkers CTX, PINP, OC, and BAP-A Systematic Review. Osteoporosis International, 30, 2381-2389. https://doi.org/10.1007/s00198-019-05132-1

[20] Szulc, P., Naylor, K., Hoyle, N.R., et al. (2017) Use of CTX-I and PINP as Bone Turnover Markers: National Bone Health Alliance Recommendations to Standardize Sample Handling and Patient Preparation to Reduce Pre-Analytical Variability. Osteoporosis International, 28, 2541-2556. https://doi.org/10.1007/s00198-017-4082-4

[21] Yang, S.W., Ma, R.J., Zhao, J.J., et al. (2018) Clinical Significance of the Ratio of Serum Beta-CTX/PINP in Multiple Myeloma Bone Diseases and Bone Metastases. Chinese Medical Journal, 98, 2583-2587.

[22] Krege, J.H., Lane, N.E., Harris, J.M., et al. (2014) PINP as a Biological Response Marker during Teriparatide Treatment for Osteoporosis. Osteoporosis International, 25, 2159-2171. https://doi.org/10.1007/s00198-014-2646-0

[23] Niimi, R., Kono, T., Nishihara, A., et al. (2014) An Algorithm Using the Early Changes in PINP to Predict the Future BMD Response for Patients Treated with Daily Teriparatide. Osteoporosis International, 25, 377-384.

https://doi.org/10.1007/s00198-013-2426-2

[24] Bhattoa, H.P., Cavalier, E., Eastell, R., et al. (2021) Analytical Considerations and Plans to Standardize or Harmonize Assays for the Reference Bone Turnover Markers PINP and $\beta$-CTX in Blood. Clinica Chimica Acta, 515, 16-20. https://doi.org/10.1016/j.cca.2020.12.023

[25] Center, J.R. (2017) Fracture Burden: What Two and a Half Decades of Dubbo Osteoporosis Epidemiology Study Data Reveal about Clinical Outcomes of Osteoporosis. Current Osteoporosis Reports, 15, 88-95. https://doi.org/10.1007/s11914-017-0352-5

[26] Yao, X., Xu, Z. and Du, X. (2019) PKP/PVP Combine Chemotherapy in the Treatment of Multiple Myeloma Patients with Vertebral Pathological Fractures: Minimum 3-Year Follow-Up of 108 Cases. Journal of Orthopaedic Surgery and Research, 14, 42. https://doi.org/10.1186/s13018-019-1078-0

[27] Zhou, T., Lin, H., Wang, H., et al. (2017) Comparative Study on the Biomechanics between Improved PVP and Traditional PKP in the Treatment of Vertebral Peripheral Wall Damage-Type OVCF. Experimental and Therapeutic Medicine, 14, 575-580. https://doi.org/10.3892/etm.2017.4542

[28] Lavelle, W.F. and Cheney, R. (2006) Recurrent Fracture after Vertebral Kyphoplasty. The Spine Journal, 6, 488-493. https://doi.org/10.1016/j.spinee.2005.10.013 
[29] Bahşi, R., Atmiş, V., Turgut, T., et al. (2020) May Zoledronic Acid Have Negative Effects on Cognition and Muscle Performance? Irish Journal of Medical Science, 189, 191-196. https://doi.org/10.1007/s11845-019-02086-5

[30] Ouyang, Z., Li, H., Zhai, Z., et al. (2018) Zoledronic Acid: Pleiotropic Anti-Tumor Mechanism and Therapeutic Outlook for Osteosarcoma. Current Drug Targets, 19, 409-421. https://doi.org/10.2174/1573399811666150615145409

[31] Coleman, R., Burkinshaw, R., Winter, M., et al. (2011) Zoledronic Acid. Expert Opinion on Drug Safety, 10, 133-145. https://doi.org/10.1517/14740338.2011.540387

[32] Greenspan, S.L., Vujevich, K., Britton, C., et al. (2018) Teriparatide for Treatment of Patients with Bisphosphonate-Associated Atypical Fracture of the Femur. Osteoporosis International, 29, 501-506. https://doi.org/10.1007/s00198-017-4286-7

[33] Lindsay, R., Krege, J.H., Marin, F., et al. (2016) Teriparatide for Osteoporosis: Importance of the Full Course. Osteoporosis International, 27, 2395-2410. https://doi.org/10.1007/s00198-016-3534-6

[34] Saag, K.G., Shane, E., Boonen, S., et al. (2007) Teriparatide or Alendronate in Glucocorticoid-Induced Osteoporosis. The New England Journal of Medicine, 357, 2028-2039. https://doi.org/10.1056/NEJMoa071408

[35] Murphy, P.Z., Iranikhah, M., Deas, C.M., et al. (2018) Fracture Risk Following Discontinuation of Teriparatide: A Review of the Literature. Consultant Pharmacist, 33, 365-375. https://doi.org/10.4140/TCP.n.2018.365

[36] Cosman, F. (2014) Anabolic and Antiresorptive Therapy for Osteoporosis: Combination and Sequential Approaches. Current Osteoporosis Reports, 12, 385-395. https://doi.org/10.1007/s11914-014-0237-9

[37] Leder, B.Z. (2018) Optimizing Sequential and Combined Anabolic and Antiresorptive Osteoporosis Therapy. JBMR Plus, 2, 62-68. https://doi.org/10.1002/jbm4.10041

[38] Suzuki, H. and Takeuchi, Y. (2012) Combination or Sequential Treatment Using PTH and Anti-Resorption Therapies. Clinical Calcium, 22, 415-420.

[39] Diab, D.L. and Watts, N.B. (2013) Postmenopausal Osteoporosis. Current Opinion in Endocrinology, Diabetes and Obesity, 20, 501-509. https://doi.org/10.1097/01.med.0000436194.10599.94

[40] Vasikaran, S.D., Bhattoa, H.P., Eastell, R., et al. (2020) Harmonization of Commercial Assays for PINP; the Way Forward. Osteoporosis International, 31, 409-412. https://doi.org/10.1007/s00198-020-05310-6 